\title{
On the hydrogen-like atom in five-dimensional space
}

\author{
Le Van Hoang, Tony J Viloria and Le anh Thu \\ Department of Physics, Byelorussian State University, Minsk 220080, USSR
}

Received 24 October 1990, in final form 5 March 1991

\begin{abstract}
We propose an easy and effective variant of the realization of the dynamical symmetry of hydrogen-like atoms in five-dimensional space, based on the relationship between the Schrödinger equation for the isotropic harmonic oscillator in four-dimensional complex space and the Schrödinger equation for the one-electron atom in five-dimensional space. By the use of the path integral we also establish the relationship between the Coulomb Green function and the Green function for the isotropic harmonic oscillator in fourdimensional complex space.
\end{abstract}

\section{Introduction}

Komarov and Romanova (1982) found a connection between the Schrödinger equation for a hydrogen-like atom and that for an isotropic harmonic oscillator in twodimensional complex space and from this suggested an easy and effective variant for the realization of the dynamic symmetry of the hydrogen atom, that allows algebraic methods to be used in the solution of many problems of the electron's motion in the Coulomb and electromagnetic fields (see, for example, Komarov et al (1987)). The probable connection between the Schrödinger equation for a hydrogen atom and that for an isotropic harmonic oscillator in four-dimensional real space was examined long ago (see, for example, Bergmann and Frishman (1965)) and has been discussed from different points of view in other papers (see, for example, Chen 1982, Cornish 1984, Kibler and Negadi 1983), which shows its great significance. In this paper, based on the idea of Komarov and Romanova (1982), the connection between the Schrödinger equation for a hydrogen atom in five-dimensional space and that for an isotropic harmonic oscillator in four-dimensional complex spacet is established. Using this connection it is shown that in the five-dimensional hydrogen atom, the algebraic method can also be used (section 3). Developing this idea further in section 4 the connection between the Coulomb Green function in five-dimensional space and the Green function for an isotropic harmonic oscillator is established.

† The relationship between the Schrödinger equation for the hydrogen atom in the five-dimensional space and that for the oscillator in eight-dimensional space was first developed by Davtyan et al (1987). However, from our point of view, the relationship built by us is more suitable for the realization of algebraic methods. 


\section{The connection between the Schrödinger equations}

First of all let us define a closed system of 16 four-row matrices in the following way:

$$
\begin{array}{lc}
I=\text { unit matrix } & \Gamma_{1}=\left(\begin{array}{cc}
\sigma_{1} & 0 \\
0 & \sigma_{1}
\end{array}\right) \quad \Gamma_{2}=\left(\begin{array}{cc}
\sigma_{2} & 0 \\
0 & \sigma_{2}
\end{array}\right) \\
\Gamma_{3}=\left(\begin{array}{cc}
0 & \sigma_{3} \\
\sigma_{3} & 0
\end{array}\right) \quad \Gamma_{4}=\left(\begin{array}{cc}
0 & -\mathrm{i} \sigma_{3} \\
\mathrm{i} \sigma_{3} & 0
\end{array}\right) \quad \Gamma_{5}=\left(\begin{array}{cc}
-\sigma_{3} & 0 \\
0 & \sigma_{3}
\end{array}\right) \\
\Gamma_{[\mu, \nu]}=\frac{1}{2} \mathrm{i}\left(\Gamma_{\mu} \Gamma_{\nu}-\Gamma_{\nu} \Gamma_{\mu}\right) \quad(\mu, \nu=1, \ldots, 5)
\end{array}
$$

where the $\sigma_{k}$ are Pauli matrices.

Direct calculations show the generation of a closed algebra and the Hermiticity of the matrix system (1), which responds to the following properties:

$$
\begin{aligned}
& \Gamma_{\nu}^{+}=\Gamma_{\nu} \quad \Gamma_{[\nu, \mu]}^{+}=\Gamma_{[\nu, \mu]} \quad \Gamma_{\nu} \Gamma_{\mu}+\Gamma_{\mu} \Gamma_{\nu}=2 \delta_{\nu \mu} \\
& {\left[\Gamma_{\nu}, \Gamma_{\mu}\right]=-2 \mathrm{i} \Gamma_{[\nu, \mu]} \quad\left[\Gamma_{\nu}, \Gamma_{[\lambda, \mu]}\right]=2 \mathrm{i} \delta_{\nu \lambda} \Gamma_{\mu}-2 \mathrm{i} \delta_{\nu \mu} \Gamma_{\lambda}} \\
& {\left[\Gamma_{[\nu, \mu]}, \Gamma_{[\lambda, \sigma]}\right]=2 \mathrm{i} \delta_{\mu \lambda} \Gamma_{[\nu, \sigma]}-2 \mathrm{i} \delta_{\nu \lambda} \Gamma_{[\mu, \sigma]}+2 \mathrm{i} \delta_{\nu \sigma} \Gamma_{[\mu, \lambda]}-2 \mathrm{i} \delta_{\mu \sigma} \Gamma_{[\nu, \lambda]}}
\end{aligned}
$$

where the plus sign denotes the Hermitian conjugate operation with summation over repeated indices.

Now, let us consider the Schrödinger equation for an isotropic harmonic oscillator in four-dimensional complex space with the coordinates $\xi_{s}(s=1, \ldots, 4)$ :

$$
\begin{aligned}
& \hat{\mathscr{H}} \psi(\xi)=z \psi(\xi) \\
& \hat{\mathscr{H}}=-\frac{1}{2} \frac{\partial^{2}}{\partial \xi_{s} \partial \xi_{s}^{*}}+\frac{1}{2} \omega^{2} \xi_{s} \xi_{s}^{*} .
\end{aligned}
$$

Here the asterisk denotes the complex conjugate operation; $\omega$ is a real positive number; $z$ is the eigenvalue of the operator $\hat{\mathscr{H}}$. Operator $\hat{\mathscr{H}}(4)$ commutes with the following operators:

$$
\begin{aligned}
& \hat{Q}_{1}=\frac{1}{2}\left(\xi_{s}^{*} \frac{\partial}{\partial \xi_{s}^{*}}-\xi_{s} \frac{\partial}{\partial \xi_{s}}\right) \\
& \hat{Q}_{2}=\frac{1}{2} \mathrm{i}\left(\Gamma_{2} \Gamma_{4}\right)_{t s}\left(\xi_{s}^{*} \frac{\partial}{\partial \xi_{t}}+\xi_{s} \frac{\partial}{\partial \xi_{t}^{*}}\right) \\
& \hat{Q}_{3}=\frac{1}{2}\left(\Gamma_{2} \Gamma_{4}\right)_{r s}\left(\xi_{s}^{*} \frac{\partial}{\partial \xi_{t}}-\xi_{s} \frac{\partial}{\partial \xi_{t}^{*}}\right)
\end{aligned}
$$

which generate a closed algebra. It follows from the commutation relationships for the operators (5) that

$$
\left[\hat{Q}_{i}, \hat{Q}_{j}\right]=\mathrm{i} \varepsilon_{i j k} \hat{Q}_{k} \quad(i, j, k=1, \ldots, 3) .
$$

The scalar product of the wavefunction in the $\xi$-space can be defined as

$$
\langle\varphi(\xi) \mid \psi(\xi)\rangle=\int \prod_{s=1}^{4} \mathrm{~d} \xi_{s}^{\prime} \int \prod_{i=1}^{4} \mathrm{~d} \xi_{t}^{\prime \prime} \varphi^{*}\left(\xi^{\prime}, \xi^{\prime \prime}\right) \psi\left(\xi^{\prime}, \xi^{\prime \prime}\right)
$$


where $\xi_{s}^{\prime}=\operatorname{Re} \xi_{s}, \xi_{s}^{\prime \prime}=\operatorname{Im} \xi_{s}$. Thus operators $\hat{H}, \hat{Q}_{1}, \hat{Q}_{2}$ and $\hat{Q}_{3}$ are Hermitian with respect to the scalar product (7). Hence equation (3) can be written as follows.

$$
\begin{aligned}
& \tilde{\mathscr{H}} \tilde{\psi}(\xi)=-\frac{1}{2} \omega^{2} \tilde{\psi}(\xi) \\
& \tilde{\mathscr{H}}=-\frac{1}{2 \xi_{s} \xi_{s}^{*}} \frac{\partial^{2}}{\partial \xi_{t} \partial \xi_{t}^{*}}-\frac{z}{\xi_{s} \xi_{s}^{*}}
\end{aligned}
$$

so $-\frac{1}{2} \omega^{2}$ can be regarded as an eigenvalue of operator $\tilde{\mathscr{H}}$. For operator $\tilde{\mathscr{H}}$ to be Hermitian, the definition of the scalar product for the wavefunction must be changed:

$$
\langle\tilde{\varphi}(\xi) \mid \tilde{\psi}(\xi)\rangle=\frac{32}{\pi^{2}} \int \prod_{s=1}^{4} \mathrm{~d} \xi_{s}^{\prime} \int \prod_{t=1}^{4} \mathrm{~d} \xi_{t}^{\prime \prime} \tilde{\varphi}^{*}\left(\xi^{\prime}, \xi^{\prime \prime}\right) \tilde{\psi}\left(\xi^{\prime}, \xi^{\prime \prime}\right) \xi_{u}^{*} \xi_{u} .
$$

Let us now make a substitution of the variables in equation (8) using the following correlation:

$$
\begin{aligned}
& x_{\lambda}=\xi_{s}^{*}\left(\Gamma_{\lambda}\right)_{s t} \xi_{t} \quad \lambda=1, \ldots, 5 \\
& \phi_{1}=-\frac{\mathrm{i}}{2} \ln \left(\xi_{1} \xi_{4} / \xi_{1}^{*} \xi_{4}^{*}\right) \\
& \phi_{2}=-\frac{i}{2} \ln \left(\xi_{i}^{*} \xi_{4} / \xi_{1} \xi_{4}^{*}\right) \\
& \phi_{3}=\arctan \left(2 \sqrt{\xi_{1} \xi_{1}^{*} \xi_{4} \xi_{4}^{*}} /\left(\xi_{4} \xi_{4}^{*}-\xi_{1} \xi_{1}^{*}\right)\right) \\
& \frac{32}{\pi^{2}} \prod_{s=1}^{4} \mathrm{~d} \xi_{s}^{\prime} \prod_{i=1}^{4} \mathrm{~d} \xi_{i}^{\prime \prime} \xi_{u} \xi_{u}^{*}=\frac{1}{8 \pi^{2}} \sin \phi_{3} \mathrm{~d} \phi_{1} \mathrm{~d} \phi_{2} \mathrm{~d} \phi_{3} \prod_{k=1}^{5} \mathrm{~d} x_{k} .
\end{aligned}
$$

The properties of the variables $\xi_{s}$ are defined in such a way that the $x_{\lambda}$ are components of the five-dimensional real vector $r$. After the substitution of (11), (8) takes the form:

$$
\begin{gathered}
\left\{-\frac{1}{2}\left[\Delta+\frac{1}{r} \frac{\partial^{2} \phi_{k}}{\partial \xi_{s} \partial \xi_{s}^{*}} \frac{\partial}{\partial \phi_{k}}+\frac{1}{r}\left(\frac{\partial \phi_{k}}{\partial \xi_{s}} \frac{\partial x_{i}}{\partial \xi_{s}^{*}}+\frac{\partial x_{i}}{\partial \xi_{s}} \frac{\partial \phi_{k}}{\partial \xi_{s}^{*}}\right) \frac{\partial^{2}}{\partial x_{i} \partial \phi_{k}}\right.\right. \\
\left.\left.+\frac{1}{r} \frac{\partial \phi_{k}}{\partial \xi_{s}} \frac{\partial \phi_{l}}{\partial \xi_{s}^{*}} \frac{\partial^{2}}{\partial \phi_{k} \partial \phi_{l}}\right]-\frac{z}{r}\right\} \tilde{\psi}(\boldsymbol{r}, \phi) \\
=E \tilde{\psi}(\boldsymbol{r}, \phi)
\end{gathered}
$$

and the scalar product of the wavefunction (10) can be rewritten:

$$
\langle\tilde{\varphi} \mid \tilde{\psi}\rangle=\frac{1}{8 \pi^{2}} \int \mathrm{d} \boldsymbol{r} \int_{0}^{2 \pi} \mathrm{d} \phi_{1} \int_{0}^{2 \pi} \mathrm{d} \phi_{2} \int_{0}^{\pi} \mathrm{d} \phi_{3} \sin \phi_{3} \tilde{\varphi}^{*}(\boldsymbol{r}, \phi) \tilde{\psi}(\boldsymbol{r}, \phi)
$$

In equation (12)

$$
r=\sqrt{x_{\lambda} x_{\lambda}} \quad \Delta=\frac{\partial^{2}}{\partial x_{\lambda} \partial x_{\lambda}} \quad \lambda=1, \ldots, 5 .
$$

From (12) and (13) it follows that the functions $\tilde{\psi}$, which do not depend on the variables $\phi_{i}$, are solutions of the Schrödinger equation for the five-dimensional hydrogen atom 
with negative energy, if $E=-\frac{1}{2} \omega^{2}$ and $z$ is the nuclear charge. From the substitution of variables (11) the following correlation can be obtained

$$
\begin{aligned}
& \hat{Q}_{1}=\mathrm{i} \frac{\partial}{\partial \phi_{1}} \\
& \hat{Q}_{2}=-\mathrm{i} \frac{\sin \phi_{1}}{\tan \phi_{3}} \frac{\partial}{\partial \phi_{1}}+\mathrm{i} \frac{\sin \phi_{1}}{\sin \phi_{3}} \frac{\partial}{\partial \phi_{2}}+\mathrm{i} \cos \phi_{1} \frac{\partial}{\partial \phi_{3}} \\
& \hat{Q}_{3}=-\mathrm{i} \frac{\cos \phi_{1}}{\tan \phi_{3}} \frac{\partial}{\partial \phi_{1}}-\mathrm{i} \frac{\cos \phi_{1}}{\sin \phi_{3}} \frac{\partial}{\partial \phi_{2}}-\mathrm{i} \sin \phi_{1} \frac{\partial}{\partial \phi_{3}} .
\end{aligned}
$$

System (14) represents a linear form with respect to the operators $\partial / \partial \phi_{i}$ and determinant (i/sin $\phi_{3}$ ) which does not equal zero. From this it follows that all wavefunctions which are independent of $\phi_{i}$ belong to the zero eigenvalue of operators $\hat{Q}_{i}$ and vice versa. Therefore we have that the solutions of equation (3), which belong to the zero eigenvalue of operators $\hat{Q}_{i}(i=1,2,3)$ are solutions of the Schrödinger equation for the hydrogenlike atom in five-dimensional space.

\section{Algebraic method in the solution of the Schrödinger equation}

Now we will show that the found connection will enable us to build the solution of the Schrödinger equation for the hydrogen atom in five-dimensional space by purely algebraic methods.

Let us define the operators:

$$
\begin{aligned}
& a_{s}(\omega)=\sqrt{\frac{\omega}{2}}\left(\xi_{s}+\frac{1}{\omega} \frac{\partial}{\partial \xi_{s}^{*}}\right) \\
& b_{s}(\omega)=\sqrt{\frac{\omega}{2}}\left(\xi_{s}^{*}+\frac{1}{\omega} \frac{\partial}{\partial \xi_{s}}\right)
\end{aligned}
$$

and the Hermitian conjugate operators for them (relative to the scalar product for the wavefunctions (7)):

$$
\begin{aligned}
& a_{s}^{+}(\omega)=\sqrt{\frac{\omega}{2}}\left(\xi_{s}^{*}-\frac{1}{\omega} \frac{\partial}{\partial \xi_{s}}\right) \\
& b_{s}^{+}(\omega)=\sqrt{\frac{\omega}{2}}\left(\xi_{s}-\frac{1}{\omega} \frac{\partial}{\partial \xi_{s}^{*}}\right)
\end{aligned}
$$

where $\omega$ is a real positive number.

These operators satisfy the commutation relationships:

$$
\left[a_{s}(\omega), a_{t}^{+}(\omega)\right]=\delta_{s i} \quad\left[b_{s}(\omega), b_{t}^{+}(\omega)\right]=\delta_{s t}
$$

(we have oniy included non-zero commutators).

Using (15)-(17) we find that

$$
\hat{\mathscr{H}}=\frac{\omega}{2}\left(a_{s}^{+} a_{s}+b_{s}^{+} b_{s}+4\right)
$$


and

$$
\begin{aligned}
& \hat{Q}_{1}=\frac{1}{2}\left(a_{s}^{+} a_{s}-b_{s}^{+} b_{s}\right) \\
& \hat{Q}_{2}=\frac{\mathrm{i}}{2}\left(\Gamma_{2} \Gamma_{4}\right)_{t s}\left(a_{s}^{+} b_{t}+b_{s}^{+} a_{t}\right) \\
& \hat{Q}_{2}=\frac{1}{2}\left(\Gamma_{2} \Gamma_{4}\right)_{t s}\left(a_{s}^{+} b_{t}-b_{s}^{+} a_{t}\right) .
\end{aligned}
$$

The expressions (15)-(19) clearly make a set of solutions for the equation:

$$
\hat{\mathscr{H}}|\psi\rangle=z|\psi\rangle \text {. }
$$

The state-vector (in non-normalized form)

$$
|\psi\rangle=a_{s_{1}}^{+} a_{s_{2}}^{+} \ldots a_{s_{n_{a}}}^{+} b_{t_{1}}^{+} b_{z_{2}}^{+} \ldots b_{t_{n_{b}}}^{+}|0\rangle
$$

( $n_{a}$ and $n_{b}$ are positive numbers) is the solution of equation (20), which belongs to the eigenvalue:

$$
z=\frac{\omega}{2}\left(n_{a}+n_{b}+4\right)
$$

The vacuum state in (21) is determined by the following equations:

$$
a_{s}|0\rangle=b_{s}|0\rangle=0
$$

and the condition of normalization is: $\langle 0(\omega) \mid 0(\omega)\rangle=1$.

From the set of vectors (21) comes the vector $|\tilde{\psi}\rangle$ which belongs to the zero eigenvalue of operators $\hat{Q}_{i}$, i.e. it satisfies the following equations

$$
\begin{aligned}
& \hat{Q}_{1}|\tilde{\psi}\rangle=0 \\
& \hat{Q}_{2}|\tilde{\psi}\rangle=0 \\
& \hat{Q}_{3}|\tilde{\psi}\rangle=0 .
\end{aligned}
$$

It is easy to deduce that condition (24) demands that, in $|\tilde{\psi}\rangle$, the number of creation operators $a^{+}$should be equal to the number of creation operators $b^{+}: n_{a}=n_{b}$, as

$$
\hat{Q}_{1}|\tilde{\psi}\rangle=\left(n_{a}-n_{b}\right)|\tilde{\psi}\rangle \text {. }
$$

Hence we demand the following solution:

$$
|\tilde{\psi}\rangle=\prod_{k}\left(\alpha_{i j}^{k} a_{i}^{+} b_{j}^{+}\right)|0\rangle
$$

If we substitute (27) into (25) and (26) and use (17), we find that they are satisfied if

$$
\left[\hat{Q}_{2}, \alpha_{i j}^{k} a_{i}^{+} b_{j}^{+}\right]=\left[\hat{Q}_{3}, \alpha_{i j}^{k} a_{i}^{+} b_{j}^{+}\right]=0
$$

provided the matrices $\alpha^{k} \Gamma_{2} \Gamma_{4}$ and $\Gamma_{2} \Gamma_{4} \alpha^{k}$ are antisymmetric. Using the properties of the matrices $\Gamma(2)$ it can be easily proved that the matrices which obey conditions (28) are the matrices: $I, \Gamma_{j}(j=1, \ldots, 5)$. Finally the solution of equation (20), taking conditions (24), (25) and (26) into account, can be introduced as follows.

$$
\left|\psi\left(n, \omega_{n}\right)\right\rangle=\left(M^{+}\right)^{n_{0}}\left(m_{1}^{+}\right)^{n_{1}}\left(m_{2}^{+}\right)^{n_{2}}\left(m_{3}^{+}\right)^{n_{3}}\left(m_{4}^{+}\right)^{n_{4}}\left(m_{5}^{+}\right)^{n_{5}}\left|0\left(\omega_{n}\right)\right\rangle .
$$

Here we introduce the notation:

$$
M^{+}=a_{s}^{+} b_{s}^{+} \quad m_{\lambda}^{+}=\left(\Gamma_{\lambda}\right)_{s t} a_{s}^{+} b_{t}^{+} \quad n=n_{0}+n_{1}+\ldots+n_{5}=0,1,2, \ldots
$$


From the following formulation of the relationship it can be concluded that the non-normalized vector (29), which represents a solution of (12) which is independent of the variables $\phi_{i}$, is also a solution of the Schrödinger equation for the hydrogen atom in five-dimensional space. The energy levels are represented by the equation

$$
E_{n}=-\frac{1}{2} \frac{z^{2}}{(n+2)^{2}} \quad \omega_{n}=\frac{z}{n+2} .
$$

The energy spectrum for the five-dimensional hydrogen atom (31) coincides with the one first found by Levy (1950). Let us examine the matrix elements of the operators. Matrix elements of the operators $\hat{A}$ acting in $r$-space, are found, as shown later, after transformation of operators $r \hat{A}$ into $\xi$-space, by means of the expression

$$
\int \mathrm{d} \boldsymbol{r} \psi_{n}^{*}(\boldsymbol{r}) \hat{A} \psi_{m}(\boldsymbol{r})=\left\langle\psi\left(n, \omega_{n}\right)|r \hat{A}| \psi\left(m, \omega_{m}\right)\right\rangle
$$

where the state vector $\left|\psi\left(n, \omega_{n}\right)\right\rangle$, corresponding to the wavefunction $\psi_{n}(\boldsymbol{r})$ of the discrete spectrum of the hydrogen atom in five-dimensional space, is determined by equation (29) and the condition of normalization

$$
\left\langle\psi\left(m, \omega_{m}\right)|r| \psi\left(m, \omega_{m}\right)\right\rangle=1 .
$$

Expression (32) allows us to perform the calculations algebraically without using the explicit form of the wavefunctions in the coordinate representation. We start with a very simple unitary transformation to carry out the transition from one frequency to another

$$
\begin{aligned}
& \left|\psi\left(\omega^{\prime}\right)\right\rangle=U\left(\omega^{\prime}, \omega\right)|\psi(\omega)\rangle \\
& a_{s}\left(\omega^{\prime}\right)=U\left(\omega^{\prime}, \omega\right) a_{s}(\omega) U^{-1}\left(\omega^{\prime}, \omega\right)
\end{aligned}
$$

where

$$
U\left(\omega^{\prime}, \omega\right)=\exp \left\{\ln \sqrt{\frac{\omega^{t}}{\omega}}\left[a_{s}(\omega) b_{s}(\omega)-a_{s}^{+}(\omega) b_{s}^{+}(\omega)\right]\right\}
$$

or in the normal form:

$$
\begin{aligned}
U\left(\omega^{\prime}, \omega\right)=\exp & {\left[\frac{\omega-\omega^{\prime}}{\omega+\omega^{\prime}} M^{+}(\omega)\right] \exp \left[-(N(\omega)+4) \ln \frac{\omega^{\prime}+\omega}{2 \sqrt{\omega \omega^{\prime}}}\right] } \\
& \times \exp \left[\frac{\omega-\omega^{\prime}}{\omega+\omega^{\prime}} M(\omega)\right]
\end{aligned}
$$

where

$$
N(\omega)+4=a_{s}^{+}(\omega) a_{s}(\omega)+b_{s}^{+}(\omega) b_{s}(\omega)+4 .
$$

Second, by transforming the operators $\hat{A}$ into $\xi$-space and then into the operators $a_{s}\left(a_{s}^{+}\right)$and $b_{s}\left(b_{s}^{+}\right)$, the operators will only appear in the following combinations:

$$
\begin{aligned}
& M(\omega)=a_{s}(\omega) b_{s}(\omega) \quad M^{+}(\omega)=a_{s}^{+}(\omega) b_{s}^{+}(\omega) \\
& N(\omega)=a_{s}^{+}(\omega) a_{s}(\omega)+b_{s}^{+}(\omega) b_{s}(\omega) \\
& m_{\lambda}(\omega)=\left(\Gamma_{\lambda}\right)_{s t} a_{t}(\omega) b_{s}(\omega) \quad m_{\lambda}^{+}(\omega)=\left(\Gamma_{\lambda}\right)_{s t} a_{s}^{+}(\omega) b_{t}^{+}(\omega) \\
& n_{\lambda}(\omega)=\left(\Gamma_{\lambda}\right)_{s t}\left[a_{s}^{+}(\omega) a_{t}(\omega)+b_{t}^{+}(\omega) b_{s}(\omega)\right] \\
& n_{[\lambda, \mu]}(\omega)=\left(\Gamma_{\left[\lambda_{t} \mu\right]}\right)_{s t}\left[a_{s}^{+}(\omega) a_{t}(\omega)-b_{t}^{+}(\omega) b_{s}(\omega)\right] .
\end{aligned}
$$


The 28 operators in (37) generate a closed algebra. By setting up the linear combinations from the operators (37)

$$
\begin{array}{ll}
L_{0 \lambda}=\frac{1}{2} n_{\lambda} \quad L_{\lambda \mu}=-\frac{1}{2} n_{[\lambda, \mu]} \quad(\lambda, \mu=1, \ldots, 5) \\
L_{06}=\frac{1}{2}\left(M-M^{+}\right) & L_{07}=-\frac{1}{2} \mathrm{i}\left(M+M^{+}\right) \\
L_{\lambda 6}=\frac{1}{2} \mathrm{i}\left(m_{\lambda}+m_{\lambda}^{+}\right) & L_{\lambda 7}=\frac{1}{2}\left(m_{\lambda}-m_{\lambda}^{+}\right) \\
L_{67}=-\frac{1}{2}(N+4) &
\end{array}
$$

and introducing the definition

$$
L_{k l}=-L_{l k} \quad(k, l=0, \ldots, 7)
$$

we obtain, with the help of (17), the following

$$
\left[L_{k l}, L_{m n}\right]=\mathrm{i}\left(\delta_{k m} L_{l n}+\delta_{l n} L_{k m}-\delta_{k n} L_{l m}-\delta_{l m} L_{k n}\right) \text {. }
$$

Thus we find that the algebra of operators (37) is the Lie algebra of the group $\operatorname{SO}(6,2)$ (see, for example, Malkin and Manko (1979)), which is the dynamical symmetry group of the Schrödinger equation for the five-dimensional hydrogen-like atom. As in Komarov et al (1982) we note that transformation (36) permits us to calculate the matrix elements of the operators, between the state-vectors, which are connected with different values of the charge in equation (12).

\section{The connection between the Green functions}

The Green function for (3) and (4) in the 'energy' representation is a solution of the following equation:

$$
\left(z+\frac{1}{2} \frac{\partial^{2}}{\partial \xi_{s} \partial \xi_{s}^{*}}-\frac{1}{2} \omega^{2} \xi_{s} \xi_{s}^{*}\right) U(\xi, \eta ; z)=\mathrm{i} \prod_{s=1}^{4} \delta\left(\xi_{s}^{\prime}-\eta_{s}^{\prime}\right) \prod_{t=1}^{4} \delta\left(\xi_{t}^{\prime \prime}-\eta_{t}^{\prime \prime}\right)
$$

where $\xi_{s}^{\prime}=\operatorname{Re} \xi_{s}, \xi_{s}^{\prime \prime}=\operatorname{Im} \xi_{s}$ and $\delta(x)$ is a Dirac $\delta$-function. One of the ways of constructing the function $U(\xi, \eta ; z)$ is to represent it as a path integral (see, for example, Slavnov and Fadeev (1978)):

$U(\xi, \eta ; z)=\int_{0}^{\infty} \mathrm{d} \theta \mathrm{e}^{\mathrm{i} z \theta} \prod_{\theta^{\prime}} D^{8} \xi\left(\theta^{\prime}\right) \exp \left\{\mathrm{i} \int_{0}^{\theta} \mathrm{d} \theta^{\prime}\left[2 \dot{\xi}_{s}^{*} \dot{\xi}_{s}^{*}-\frac{1}{2} \omega^{2} \xi_{s}^{*} \xi_{s}\right]\right\}$

where $\dot{\xi}_{s}\left(\theta^{\prime}\right)=\mathrm{d} \xi_{s}\left(\theta^{\prime}\right) / \mathrm{d} \theta^{\prime}$ and $\xi_{s}(\theta)=\xi_{s}, \xi_{s}(0)=\eta_{s}$. Equation (42) is regarded as a limit (when $\varepsilon \rightarrow 0, N \rightarrow \infty,(N \varepsilon=\theta)$ ) of the following expression (see Slavnov and Fadeev (1978))

$$
\begin{aligned}
U(\xi, \eta ; z)= & \int_{0}^{\infty} \mathrm{d} \theta \lim _{\substack{\varepsilon \rightarrow 0 \\
N \rightarrow \infty}}\left(\frac{2}{\mathrm{i} \pi \varepsilon}\right)^{4 N} \prod_{k=1}^{N-1} \mathrm{~d}^{8} \xi(k) \\
& \times \exp \left\{\mathrm { i } \sum _ { k = 1 } ^ { N } \left(\frac{2}{\varepsilon}\left[\xi_{s}^{*}(k)-\xi_{s}^{*}(k-1)\right]\left[\xi_{s}(k)-\xi_{s}(k-1)\right]\right.\right. \\
& \left.\left.+z \varepsilon-\frac{1}{2} \omega^{2} \varepsilon \xi_{s}^{*}(k-1) \xi_{s}(k-1)\right)\right\}
\end{aligned}
$$


where $\xi_{s}(N)=\xi_{s}, \xi_{s}(0)=\eta_{s}$ and $\mathrm{d}^{8} \xi(k)=\mathrm{d} \xi_{1}^{\prime} \mathrm{d} \xi_{1}^{\prime \prime} \mathrm{d} \xi_{2}^{\prime} \mathrm{d} \xi_{2}^{\prime \prime} \mathrm{d} \xi_{3}^{\prime} \mathrm{d} \xi_{3}^{\prime \prime} \mathrm{d} \xi_{4}^{\prime} \mathrm{d} \xi_{4}^{\prime \prime}$ (for brevity, we use the notation $\xi_{s}(k) \equiv \xi_{s}(k \varepsilon)$ ). To establish the relationship between the function $U(\xi, \eta ; z)$ and the Coulomb Green function, we change the variables in (42), choosing as in (11) as part of the new variables:

$$
x_{\lambda}(\theta)=\left(\Gamma_{\lambda}\right)_{s t} \xi_{s}^{*}(\theta) \xi_{t}(\theta) .
$$

Making use of the relationship

$$
\left(\Gamma_{\lambda}\right)_{s t}\left(\Gamma_{\lambda}\right)_{u v}=2 \delta_{s v} \delta_{u t}-\delta_{s t} \delta_{u v}+2\left(\Gamma_{2} \Gamma_{4}\right)_{s u}\left(\Gamma_{2} \Gamma_{4}\right)_{t v}
$$

we can easily ascertain that

$$
\begin{aligned}
\dot{\xi}_{s}^{*}(\theta) \dot{\xi}_{s}(\theta)= & \frac{1}{4 r(\theta)} \dot{x}_{\lambda}(\theta) \dot{x}_{\lambda}(\theta)-\frac{1}{4 r(\theta)}\left[\dot{\xi}_{s}^{*}(\theta) \xi_{s}(\theta)-\xi_{s}^{*}(\theta) \dot{\xi}_{s}(\theta)\right]^{2} \\
& -\frac{1}{4 r(\theta)}\left[\left(\Gamma_{2} \Gamma_{4}\right)_{s t}\left(\dot{\xi}_{s}^{*}(\theta) \xi_{i}^{*}(\theta)-\xi_{t}(\theta) \dot{\xi}_{s}(\theta)\right)\right]^{2} \\
& +\frac{1}{4 r(\theta)}\left[\left(\Gamma_{2} \Gamma_{4}\right)_{s t}\left(\dot{\xi}_{s}^{*}(\theta) \xi_{i}^{*}(\theta)+\xi_{t}(\theta) \dot{\xi}_{s}(\theta)\right)\right]^{2}
\end{aligned}
$$

where

$$
\dot{x}_{\lambda}(\theta)=\mathrm{d} x_{\lambda}(\theta) / \mathrm{d} \theta \quad r(\theta)=\sqrt{x_{\lambda}(\theta) x_{\lambda}(\theta)}=\xi_{s}^{*}(\theta) \xi_{s}(\theta) .
$$

From (46) it follows that in calculating the path integral using (43), the appropriate substitution of variables is

$$
\begin{gathered}
x_{\lambda}(k)=\left(\Gamma_{\lambda}\right)_{s t} \xi_{s}^{*}(k) \xi_{t}(k) \\
x_{6}(k)=\mathrm{i}\left[\xi_{s}^{*}(k)-\xi_{s}^{*}(k-1)\right] \xi_{s}(k)-\mathrm{i} \xi_{s}^{*}(k)\left[\xi_{s}(k)-\xi_{s}(k-1)\right] \\
x_{7}(k)=\left(\Gamma_{2} \Gamma_{4}\right)_{s t}\left(\left[\xi_{s}^{*}(k)-\xi_{s}^{*}(k-1)\right] \xi_{t}^{*}(k)+\xi_{t}(k)\left[\xi_{s}(k)-\xi_{s}(k-1)\right]\right) \\
x_{8}(k)=\mathrm{i}\left(\Gamma_{2} \Gamma_{4}\right)_{s t}\left(\left[\xi_{s}^{*}(k)-\xi_{s}^{*}(k-1)\right] \xi_{t}^{*}(k)-\xi_{t}(k)\left[\xi_{s}(k)-\xi_{s}(k-1)\right]\right) \\
\mathrm{d}^{8} \xi(k)=\frac{1}{256 r^{4}(k)} \mathrm{d}^{8} x(k) \equiv \frac{1}{256 r^{4}(k)} \mathrm{d} x_{1}(k) \mathrm{d} x_{2}(k) \ldots \mathrm{d} x_{8}(k) \\
\begin{aligned}
U(\xi, \eta ; z)=\int_{0}^{\infty} \mathrm{d} \theta \lim _{\varepsilon \rightarrow 0}\left(\frac{2}{\mathrm{i} \pi \varepsilon}\right)^{4} \prod_{k=1}^{N-1} \frac{\mathrm{d}^{8} x(k)}{(2 \mathrm{i} \pi \varepsilon r(k))^{4}} \\
\quad \times \exp \left\{\mathrm { i } \sum _ { k = 1 } ^ { N } \left(\frac{1}{2 \varepsilon r(k)}\left[x_{\lambda}(k)-x_{\lambda}(k-1)\right]\left[x_{\lambda}(k)-x_{\lambda}(k-1)\right]\right.\right. \\
\left.\left.\quad+\frac{x_{6}^{2}(k)+x_{7}^{2}(k)+x_{8}^{2}(k)}{2 \varepsilon r(k)}+z \varepsilon-\frac{1}{2} \omega^{2} \varepsilon r(k-1)\right)\right\} .
\end{aligned}
\end{gathered}
$$

The separation of the variables $x_{6}(k), x_{7}(k), x_{8}(k)$ is due to the fact that the functions

$$
\begin{aligned}
& Q_{1}=\mathrm{i}\left[\dot{\xi}_{s}^{*}(\theta) \xi_{s}(\theta)-\xi_{s}^{*}(\theta) \dot{\xi}_{s}(\theta)\right] \\
& Q_{2}=\left(\Gamma_{2} \Gamma_{4}\right)_{s t}\left[\dot{\xi}_{s}^{*}(\theta) \xi_{t}^{*}(\theta)+\xi_{t}(\theta) \dot{\xi}_{s}(\theta)\right] \\
& Q_{3}=\mathrm{i}\left(\Gamma_{2} \Gamma_{4}\right)_{s t}\left[\dot{\xi}_{s}^{*}(\theta) \xi_{t}^{*}(\theta)-\xi_{t}(\theta) \dot{\xi}_{s}(\theta)\right]
\end{aligned}
$$

are the integrals of motion for the systems described by the Lagrange function

$$
\mathscr{L}=2 \dot{\xi}_{s}^{*}(\theta) \dot{\xi}_{s}(\theta)-\frac{1}{2} \omega^{2} \xi_{s}^{*}(\theta) \xi_{s}(\theta) .
$$


Integrating over the variables $x_{6}(k), x_{7}(k), x_{8}(k)(k=\overline{1, N-1})$ and taking into account that $U(\xi, \eta ; z)$ is the integral operator, which will be used, as shown in section 2 , in the class of functions depending on $x_{\lambda}$ only (i.e. one may integrate with respect to an 'extra' variables $\left.x_{6}(k), x_{7}(k), x_{8}(k)\right)$, we find

$K\left(r, r^{\prime} ;-\frac{1}{2} \omega^{2}\right)$

$$
\begin{aligned}
= & \frac{1}{256 r^{3}(N)} \int \cdot \mathrm{d} x_{6}(k) \mathrm{d} x_{7}(k) \mathrm{d} x_{8}(k) U(\xi, \eta ; z) \\
= & \int_{0}^{\infty} \mathrm{d} \theta \lim _{\substack{\varepsilon \rightarrow 0 \\
N \rightarrow \infty}} \frac{r(N)}{(2 \mathrm{i} \pi \varepsilon r(N))^{5 / 2}} \prod_{k=1}^{N-1} \frac{\mathrm{d}^{5} x(k)}{(2 \mathrm{i} \pi \varepsilon r(k))^{5 / 2}} \\
& \times \exp \left\{\mathrm { i } \sum _ { k = 1 } ^ { N } \left(\frac{1}{2 \varepsilon r(k)}\left[x_{\lambda}(k)-x_{\lambda}(k-1)\right]\left[x_{\lambda}(k)-x_{\lambda}(k-1)\right]\right.\right. \\
& \left.\left.+z \varepsilon-\frac{1}{2} \omega^{2} \varepsilon r(k-1)\right)\right\}
\end{aligned}
$$

where

$$
\boldsymbol{r}=\xi_{s}^{*}(\boldsymbol{\Gamma})_{s t} \xi_{t} \quad \boldsymbol{r}^{\prime}=\eta_{s}^{*}(\Gamma)_{s t} \eta_{t} .
$$

The weight $\left(256 r^{3}(N)\right)^{-1}$ on the integration over $x_{6}(k), x_{7}(k)$ and $x_{8}(k)$ is defined by the Jacobian of the transformation (48) and the condition of normalization of the wavefunctions (see section 2).

The last step is the change of 'time' variable: let

$$
\varepsilon(k)=\varepsilon r(k)
$$

which in the limit $\varepsilon \rightarrow 0$ is equivalent to the introduction of a new time variable

$$
t=\int_{0}^{\theta} \mathrm{d} \theta^{\prime} r\left(\theta^{\prime}\right)
$$

As a result we have

$K\left(\boldsymbol{r}, \boldsymbol{r}^{\prime} ;-\frac{1}{2} \omega^{2}\right)$

$$
=\int_{0}^{\infty} \mathrm{d} t \mathrm{e}^{-\mathrm{i}\left(\omega^{2} / 2\right) t} \prod_{\tau} \mathscr{D}^{5} x(\tau) \exp \left\{\mathrm{i} \int_{0}^{t} \mathrm{~d} \tau\left(\frac{1}{2} \dot{x}_{\lambda}(\tau) \dot{x}_{\lambda}(\tau)+z / r(\tau)\right)\right\}
$$

where $r(t)=r$ and $r(0)=r^{\prime}$. Denoting $E=-\frac{1}{2} \omega^{2}$ we obtain from (54)

$$
\left(E+\frac{1}{2} \Delta+\frac{z}{r}\right) K\left(\boldsymbol{r}, \boldsymbol{r}^{\prime} ; E\right)=\mathrm{i} \delta\left(\boldsymbol{r}-\boldsymbol{r}^{\prime}\right)
$$

( $\Delta$ is the Laplace operator), i.e. $K\left(\boldsymbol{r}, \boldsymbol{r}^{\prime} ; E\right)$ is the energy representation of the Coulomb Green function.

\section{Acknowledgment}

The authors of this paper are very grateful to Professor L I Komarov for his interest and comments on this work. 


\section{References}

Bergmann P and Frishman Y 1965 J. Math. Phys. 6 1855-6

Chen A C 1982 J. Math. Phys. 23 412-16

Chetouani L and Hammann T F 1986 J. Math. Phys. 27 2944-6

Cornish F H G 1984 J. Phys. A: Math. Gen. 17 323-7

Davtyan L S, Mardogan L G, Pogosyan G S, Sissakian A N and Ter-Antonyan V M 1987 J. Phys. A: Math. Gen. $206121-5$

Feynman R P and Hibbs A R 1968 Quantum Mechanics and Path Integrals (Moscow: Mir) Le van Hoang, Komarov L I and Romanova T S 1989 J. Phys. A: Math. Gen. 22 1543-52

Kibler M and Negadi T 1983 J. Phys. A: Math. Gen. $164265-8$

Komarov L I and Romanova T S 1982 Izv. Akad. Nauk BSSR (ser. Fis.-Mat. Nauk) 2 98-103

Komarov L I, Romanova T S and Chan Za An 1987 Izv. Akad. Nauk BSSR (ser. Fis.-Mat. Nauk) 1 90-8 Levy M 1950 Proc. R. Soc. 204145

Malkin I A and Manko V I 1979 Dynamical Symmetry and Coherent State of the Quantum Systems (Moscow: Nauka)

Slavnov A A and Fadeev L D 1978 An Introduction to the Quantum Theory of the Gauge Fields (Moscow: Nauka) 